\title{
INTERACTION PROTECTION CATHODIQUE ET BIOFILM EN MILIEU MARIN
}

\author{
J. Guezennec, E. Antoine et M. Conte \\ IFREMER. Plouzane
}

In marine environments cathodic protection using sacrificial anodes or impressed current is widely used. In aerated seawater a cathodic potential of - $800 \mathrm{mV} / \mathrm{SCE}$ is generally accepted as efficient against marine corrosion. Biofilms formed on the metallic surfaces exposed to seawater can promote localized corrosion and interfere with the cathodic polarization process. Experiments were conducted both in natural environments and with a monoculture of a marine bacterium in order to understand the relationship between cathodic protection and biofilm formation. Results indicated that, in the former case, there was no evidence that biofilms significantly affect the cathodic current demand. Conversely, in sterilized seawater inoculated with a single bacterial species, biofilm on the surface seriously affected the current demand. Furthermore, cathodic polarisation does not appear to influence bacterial attachment to the surfaces.

\section{Introduction}

La protection cathodique est largement utilisée dans le milieu marin comme moyen de prévention de la corrosion des alliages métalliques placés dans cet environnement. Un potentiel cathodique de $-800 \mathrm{mV} / \mathrm{ECS}$ est généralement considéré, en milieu aéré, comme suffisant pour protéger les structures contre la corrosion marine. Cependant, dans certaines conditions et notamment dans des milieux anoxiques ou dans les sédiments marins, la présence de micro-organismes telles les bactéries sulfato-réductrices et autres bactéries anaérobies nécessite une remise en cause de ce seuil de protection et un potentiel de $-900 \mathrm{mV} / \mathrm{ECS}$ est alors nécessaire pour éviter toute corrosion de ces structures.

Des récents travaux ont par ailleurs montré que l'utilisation de la protection cathodique comme moyen de protection des ouvrages nécessitait une bonne connaissance, à la fois physico-chimique et microbiologique des milieux considérés (Fisher et al, 1986), et que les critères de protection cathodique apparaissaient principalement dépendants des sites considérés. Par ailleurs, la production à partir d'un certain potentiel, de quantités non négligeables d'hydrogène cathodique, peut favoriser, à l'interface métal-milieu, le développement de bactéries sulfato-réductrices utilisant cet élément comme donneur d'électrons (Théréné, 1988), mettant ainsi en évidence le danger présenté par un mauvais choix du potentiel de polarisation ou encore par une polarisation incomplète ou momentanément interrompue.

En eau de mer circulante, le développement du voile biologique sur les surfaces est de nature, du fait de la présence physique ou de l'activité des micro-organismes constituant ce voile, à initier des corrosions à caractère localisé. La littérature est maintenant largement abondante à ce sujet. Les micro-organismes se fixent sur les surfaces selon des séquences 
connues et ces phénomènes d'adhésion et de fixation sont alors fonction de nombreux paramètres, dont la nature et les caractéristiques de ces surfaces. Ainsi toute modification apportée à ces surfaces, tant par la présence d'un dépôt ou par modification de certaines caractéristiques, telles des charges électrostatiques favorables ou défavorables induites par une polarisation, peut modifier l'attachement de ces micro-organismes et, par voie de conséquence, interférer sur les conséquences de ce voile.

L'objectif de ce travail était donc :

a) d'étudier dans le milieu naturel, l'interaction pouvant exister entre la formation du voile biologique sur les surfaces et celle du dépôt calco-magnésien;

b) d'établir un parallèle entre ces essais conduits en conditions naturelles et d'autres préalablement réalisés avec une monoculture bactérienne.

\section{Matériels et méthodes}

\section{Description des cellules d'essais}

Les essais ont été réalisés dans des réacteurs en verre Pyrex de 500 $\mathrm{ml}$ (fig. 1), munis d'un couvercle étanche à 5 sorties; une sortie principale permettant les connexions des échantillons et de la contre électrode en titane et des sorties annexes permettant soit l'arrivée d'eau de mer, le passage de l'électrode de référence et le prélèvement du milieu pour analyses.

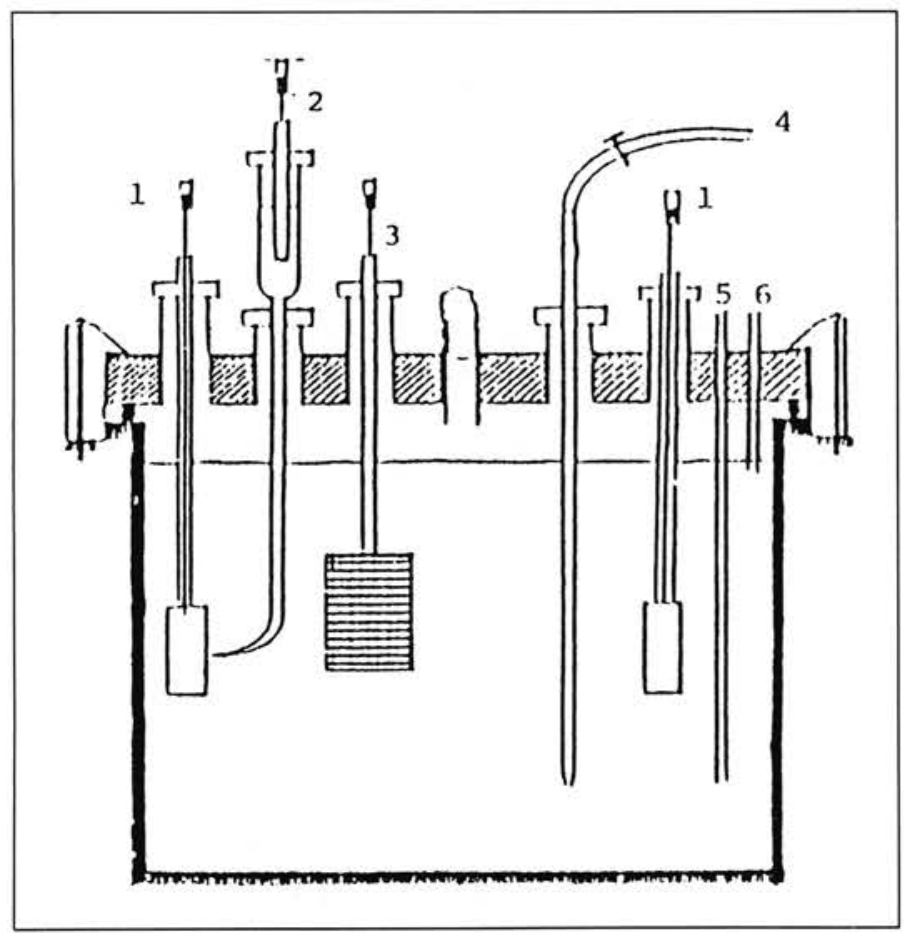

Fig. 1 - Schéma de principe du montage. 1 : Électrode de travail (6). 2 : Électrode de référence. 3 : Contre-électrode. 4 : Arrivée d'air. 5, 6 : Entrée et sortie du milieu.

Fig. 1 - Schematic diagram of the experimental system.

L'acier inoxydable utilisé était un acier 316 L. Les divers échantillons ont été préalablement polis sur une face jusqu'au papier 1000 , la soudure étant effectuée sur la face non polie. Dos et tranches de l'échantillon étaient ensuite recouverts d'une résine époxydique afin de laisser libre la seule surface polie.

Les divers échantillons ont été polarisés dans un domaine de potentiel allant de $-700 \mathrm{mV} / \mathrm{ECS}$ à $-900 \mathrm{mV} / \mathrm{ECS}$ sur une période de 8 jours. Les demandes en courant cathodique ainsi que les évolutions des potentiels d'abandon des échantillons non polarisés étaient suivis sur une centrale d'acquisition de données. Parallè- lement, une série d'échantillons a été également polarisée dans le domaine anodique à une valeur de potentiel de $0 \mathrm{mV} / \mathrm{ECS}$ soit une valeur se situant au niveau du palier de passivation de cet alliage dans le milieu considéré.

\section{Analyses microbiologiques}

Les analyses microbiologiques ont été réalisées à la fin de chaque essai. Un échantillon a alors été fixé au formaldéhyde à $2 \%$ puis coloré à l'acridine orange selon la méthode de Hobbie et al (1977). L'examen quantitatif du biofilm et le dénombrement des bactéries ont été réalisés quant à eux directement par microscopie à épifluorescence sur le coupon d'acier ainsi préparé.

\section{Analyses chimiques et biochimiques}

La biomasse bactérienne ainsi que la structure de la population bactérienne présente sur les différentes surfaces ont été suivies par analyses biochimiques et principalement par analyses des lipides membranaires bactériens. Ces analyses sont basées sur la spécificité de certains acides gras dérivés des diacyl phospholipides membranaires au seul monde procaryotique ou encore leur appartenance à un groupe ou genre bactérien (White et al, 1979. Guezennec, 1985). Après extraction des lipides, ces derniers sont préparés en trois grandes classes par chromatographie sur colonne. La phase polaire contenant les phospholipides est alors soumise à une méthanolyse acide. Après purification par chromatographie sur couche mince, les esters méthyliques d'acides gras sont analysés par chromatographie en phase gazeuse couplée à la spectrométrie de masse (Théréné, 1988).

Les acides volatils (acétate, butyrate, etc.) sont analysés directement dans la phase aqueuse par chromatographie en phase gazeuse.

\section{Résultats et discussions}

L'évolution des potentiels libres des échantillons en fonction du temps montre une remontée de ces potentiels vers des valeurs plus nobles voisines de $0 \mathrm{mV} / \mathrm{ECS}$ (fig. 2), soit des valeurs se rapprochant sensiblement du potentiel de piquration de cet acier en milieu marin, piquration observée sur un alliage en fin d'exposition et conforme aux fluctuations de potentiels notées après quelques jours d'expérimentation. Cette remontée du potentiel est à relier en grande partie, à la formation du voile biologique sur les surfaces et est fonction de nombreux facteurs physico-chimiques, hydrodynamiques et microbiologiques dont les vitesses de circulation du fluide et de colonisation des surfaces. Un exemple de courbe de polarisation obtenue après 3 jours d'exposition est représentée sur la figure 3 .

Aux potentiels cathodiques imposés, l'allure des courbes représentant les demandes en courant correspond à la formation d'un dépôt calco-magnésien sur les surfaces, conséquence de la réduction de l'oxygène et de l'alcalinisation du milieu au niveau des surfaces

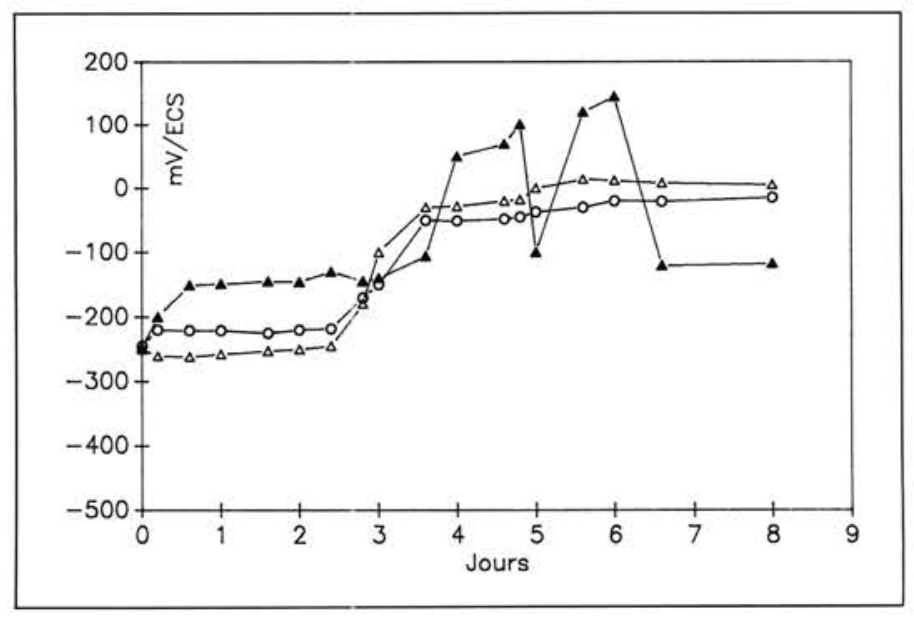

Fig. 2 - Évolution des potentiels d'abandon en eau de mer naturelle.

Fig. 2 - Open circuit potential of stainless steel samples in natural seawater. 


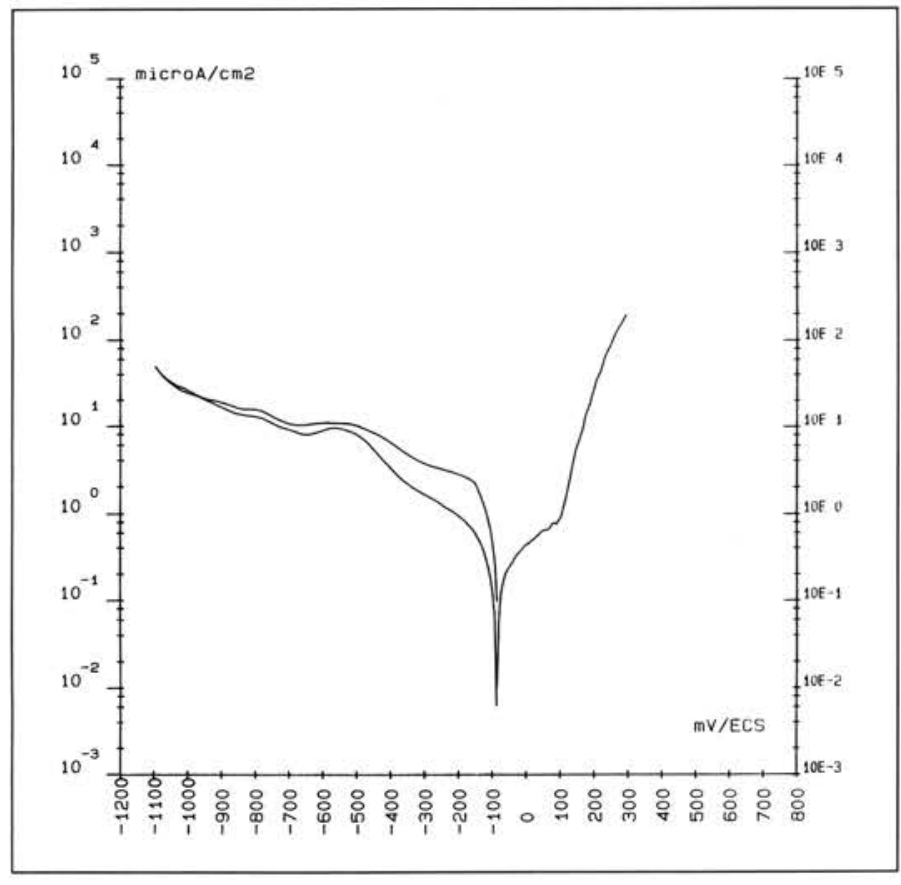

Fig. 3 - Courbe de polarisation réalisée sur un échantillon après 3 jours d'exposition en milieu naturel.

Fig. 3 - Polarization curve of stainless steel after 3 days in natural seawater.

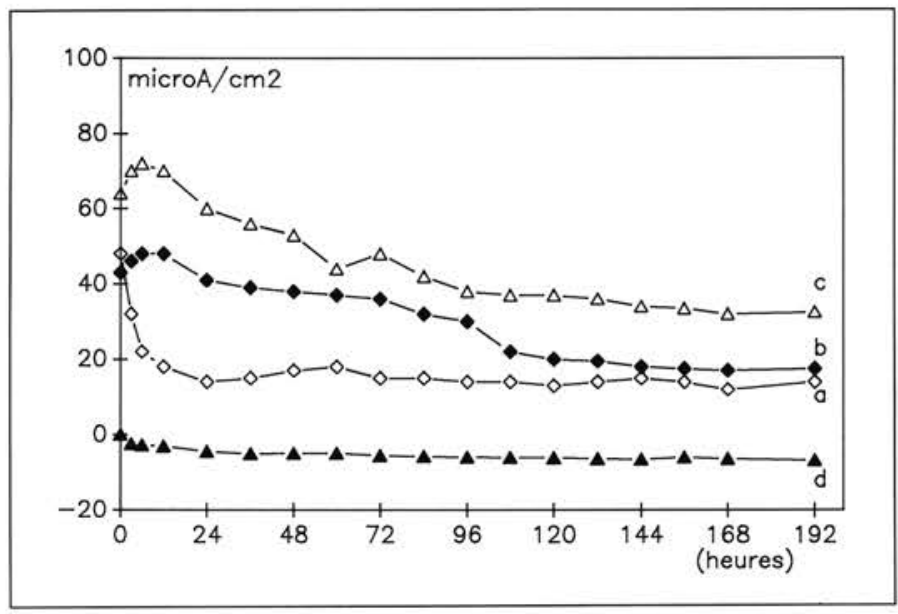

Fig. 4 - Évolution de la demande en courant cathodique. a : $-700 \mathrm{mV} / \mathrm{ECS}$ b : $-800 \mathrm{mV} / \mathrm{ECS}$ c : $-900 \mathrm{mV} / \mathrm{ECS} \mathrm{d}: 0 \mathrm{mV} / \mathrm{ECS}$

Fig. 4 - Cathodic current demand versus time.

entraînant la précipitation de sels de calcium sous forme de carbonates, et de magnésium, sous forme d'hydroxyde de magnésium. Une stabilisation de cette demande en courant est obtenue après 4 jours à des valeurs de $35 \mu \mathrm{A} / \mathrm{cm}^{2}$ et $20 \mu \mathrm{A} / \mathrm{cm}^{2}$ respectivement pour les potentiels de -900 et $-800 \mathrm{mV} / \mathrm{ECS}$ (fig. 4).

Au potentiel anodique considéré, il peut être observé une stabilisation, après 3 jours, du courant de polarisation autour d'une valeur proche de $6 \mu \mathrm{A} / \mathrm{cm}^{2}$.

Ces demandes en courant sont à rapprocher de celles obtenues lors d'expérimentations réalisées en milieu synthétique stérile (Fiskdal et Guezennec, 1988). En présence de bactéries, et plus spécifiquement d'une monoculture de Vibrio natriegens, la demande en courant cathodique est sensiblement plus importante, notamment aux potentiels de -800 et $-900 \mathrm{mV} / \mathrm{ECS}$, traduisant par ce fait l'influence de la présence de ces micro-organismes sur la protection cathodique (fig. 5). La présence d'un voile biologique sur les surfaces peut limiter la diffusion de l'oxygène vers le métal, influençant, par là même, la

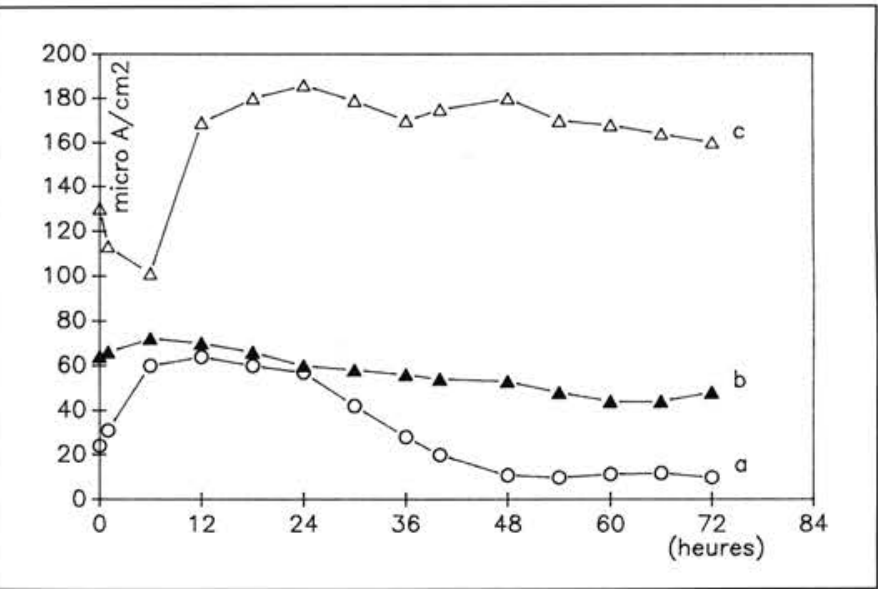

Fig. 5 - Evolution comparée de la demande en courant cathodique à - $900 \mathrm{mV} /$ ECS. a : Eau de mer stérile b : Eau de mer naturelle c : Eau de mer + Vibrio Natrigiens.

Fig. 5 - Cathodic current demand at - $900 \mathrm{mV} / \mathrm{ECS}$ in different conditions.

demande en courant cathodique. Mais il convient de tenir compte d'autres paramètres et, parmi ceux-là, l'activité métabolique des micro-organismes. L'augmentation de courant observée dans l'expérience menée en milieu stérile inoculé, correspond, à la fois, à la cinétique de formation du voile et à l'activité de ces microorganismes.

Le rapport $\mathrm{Ca} / \mathrm{Mg}$ peut être considéré comme une indication de l'effet protecteur du dépôt obtenu par polarisation cathodique. Les analyses de ce dépôt, et principalement la détermination de sa teneur en calcium et magnésium, sont représentés dans le tableau I. Dans ce même tableau, figurent les données relatives aux dépôts obtenus lors des expérimentations menées sur monoculture en milieu naturel stérilisé. Les ordres de grandeur sont totalement différents allant du $\mathrm{mg} / \mathrm{cm}^{2}$ en conditions naturelles, au $\mathrm{ng} / \mathrm{cm}^{2}$ en conditions synthétiques. Ces analyses indiquent de hautes teneurs en calcium et de faibles teneurs en magnésium confirmant la présence de carbonates de calcium comme phase cristalline majoritaire sur les surfaces. Le rapport $\mathrm{Ca} / \mathrm{Mg}$ varie cependant selon les potentiels imposés allant de $110-135$ au potentiel de $-800 \mathrm{mV} / \mathrm{ECS}$, à $45-48$ au potentiel de $-900 \mathrm{mV} / \mathrm{ECS}$. Cette différence est liée à une précipitation plus importante de sels de magnésium aux plus fortes densités de courant cathodique pour la durée d'expérimentation considérée.

Tableau I - Détermination du rapport $\mathrm{Ca} / \mathrm{Mg}$ sur les surfaces protégées cathodiquement.

Table I-Calcium and magnesium contents on the deposits formed under cathodic polarization.

\begin{tabular}{|c|c|c|c|}
\hline $\begin{array}{c}\text { Potentiel } \\
\text { cathodique }\end{array}$ & $\begin{array}{c}\text { Eau de mer } \\
\text { naturelle }\end{array}$ & $\begin{array}{c}\text { Eau de mer } \\
\text { stérile }\end{array}$ & $\begin{array}{c}\text { Eau de mer } \\
\text { + V. Natriegens }\end{array}$ \\
\hline$-700(\mathrm{mV} / \mathrm{ECS})$ & $(\mathrm{Ca})$ & 3.3 & 6.1 \\
$-800(\mathrm{mV} / \mathrm{ECS})$ & $110-135$ & 2.0 & 2.9 \\
$-900(\mathrm{mV} / \mathrm{ECS})$ & $47-48$ & 1.1 & 1.6 \\
Pot. libre & $0.5-0.6$ & 4.6 & 19 \\
\hline
\end{tabular}

En milieu eau de mer stérile inoculée, la production d'acides organiques sous forme d'acides ou de sels tels l'acétate, dans le milieu et au niveau des surfaces, est de nature à interférer avec le processus de précipitation des sels de calcium et magnésium, ou encore à initier une dissolution partielle de ce dépôt. Lors de cette expérience menée en conditions naturelles, les analyses d'acides gras volatils dans le milieu et aux différents potentiels considérés, n'ont pas mis en évidence la présence de ces acides. A l'inverse, des quantités significatives de ces acides et de leurs sels ont été déterminées lors des expérimentations avec monoculture bactérienne. 


\section{J. GUEZENNEC, E. ANTOINE, M. CONTE}

Les analyses microbiologiques réalisées au niveau des surfaces montrent, quelque soient les potentiels considérés (potentiels libres, -800 et $-900 \mathrm{mV} / \mathrm{ECS}$ et $0 \mathrm{mV} / \mathrm{ECS}$ ), des densités bactériennes comparables, comprises entre $2.810^{5}$ et $5.210^{5}$ cellules $/ \mathrm{cm}^{2}$ (tableau II). Il convient cependant de noter que la méthodologie utilisée ne permet pas de distinguer entre cellules vivantes et non vivantes présentes sur les surfaces. Ces résultats sont en accord avec ceux de Gordon et al (1981) indiquant la faible influence d'une polarisation cathodique du cuivre et du platine sur l'attachement des bactéries en milieu marin. Seule, la polarisation anodique semble pouvoir affecter cet attachement, soit du fait de la libération d'ions toxiques lors de la dissolution du métal dans le milieu (ce qui serait le cas de surfaces biologiquement actives comme le cuivre et ses alliages), soit encore par modification du $\mathrm{pH}$ interfacial. La production d' $\mathrm{H}_{2} \mathrm{O}_{2}$ à l'interface métal-solution ainsi que l'augmentation du $\mathrm{pH}$, pourraient, selon Dhar et al (1986) constituer autant de paramètres pouvant modifier l'attachement des bactéries sur les surfaces métalliques protégées cathodiquement.

Tableau II - Influence de la protection cathodique sur l'attachement des bactéries sur les surfaces.

Table II - Bacterial counts on the cathodically protected surfaces.

\begin{tabular}{|l|c|c|}
\hline $\begin{array}{c}\text { Potentiel } \\
\text { cathodique } \\
\text { (mV/ECS) }\end{array}$ & $\begin{array}{c}\text { Eau de mer } \\
\text { naturelle } \\
\text { (cellules/cm }\end{array}$ & $\begin{array}{c}\text { Eau de mer stérile } \\
+V . \text { Natrigiens } \\
\text { (cellules/cm }\end{array}$ \\
\hline-700 & $3.210^{5}$ & $4.610^{6}$ \\
-800 & $4.110^{5}$ & $2.910^{6}$ \\
-900 & $2.810^{5}$ & $1.210^{6}$ \\
Pot. libre & $5.210^{5}$ & $6.410^{6}$ \\
\hline
\end{tabular}

L'absence de différences notée lors des essais menés en eau de mer naturelle et avec monoculture semble indiquer que ces modifications apparaissent insuffisantes pour modifier l'attachement des microorganismes sur les aciers inoxydables. Des résultats identiques, tendant à montrer que la polarisation cathodique n'affecte pas, dans le domaine de protection étudié, l'attachement bactérien ont été trouvés lors d'études sur la croissance de bactéries sulfato-réductrices dans les sédiments marins (Guezennec et al, 1988) et sur l'influence de cette polarisation sur un certain nombre de souches sulfatoréductrices en milieu de culture (Guezennec et Daumas, communication personnelle).

Les analyses biochimiques basées sur l'étude des lipides dérivés des phospholipides membranaires, confirment les densités bactériennes présentes sur les surfaces polarisées et non polarisées. Ces valeurs se situent, selon les potentiels considérés, entre $2.10^{6}$ et $5.210^{6} / \mathrm{cm}^{2}$. D'un point de vue qualitatif, quelles que soient les conditions expérimentales, les bactéries présentes dans le biofilm se présentent essentiellement sous formes de bâtonnets et de coques. Ces microorganismes sont répartis de façon uniforme sur la surface et ne présentent pas d'orientations particulières. Cependant, l'ensemble de ces résultats ne signifient en rien que la polarisation cathodique ne puisse sélectionner des micro-organismes au niveau des surfaces et/ou en affecter le métabolisme. La fixation des bactéries sur les surfaces peut être affectée par de nombreux facteurs comme le pH et autres paramètres physico-chimiques, mais la résistance des micro-organismes à certaines réactions électrochimiques peut également dépendre de la physiologie de ces micro-organismes (Summers et Silver, 1978).

Enfin un fait important reste cependant les différences observées entre les expérimentations menées en milieu naturel et milieu synthétique avec monocultures, tant au niveau quantitatif qu'au niveau de la cinétique de formation du dépôt, le premier cas favorisant la formation de ce dépôt calco-magnésien, et le second la formation du voile biologique sur les surfaces.

\section{Conclusions}

L'attachement des bactéries sur les aciers inoxydables en milieu marin, ne semble pas être influencé par la mise en place d'une protection cathodique visant à protéger ces surfaces. Ces résultats obtenus pour des périodes d'expérimentation n'excédant pas 7 jours doivent cependant être reconsidérés pour des expositions plus importantes. Il apparaît, aux vues des études menées en conditions naturelles, que la mise en place d'une protection cathodique ne soit pas influencée dans un premier temps par la formation du voile biologique, ni que cette protection induise une action inhibitrice sur l'attachement bactérien.

Les différences observées entre essais réalisés en eau de mer naturelle et en milieu synthétique, tant au niveau des densités de population bactérienne que sur la nature et cinétique de formation du dépôt calco-magnésien, illustrent une nouvelle fois, les risques que peuvent comporter l'extrapolation d'essais de laboratoires au milieu réel.
H. P. Dhar, H. L. Lewis et J. O'M. Bockris, (1981), Can. J. Microbiol. Vol. 27, p. 998.

K. P. Fisher, (1981), Mat Perf. Vol. 20 no 10, p. 41.

L. Fiskdal et J. GuezenNeC, (1988), Proc 7 th Congress on Marine Corrosion and Fouling. Valence, Espagne.

A. S. Gordon, S. M. Gerchakov et L. Udey, (1981), Can. J. Microbiol. Vol. 27, p. 698.

J. GuezenneC, (1985), Thèse Doctorat Université, Paris VI.
J. Guezennec et M. Théréné, (1988), First Eur. Fed. of Corrosion Workshop on Microbial Corrosion, p. 93.

J. E. Hobbie, R. J. Daley et S. JASPER, (1977), Appl Environ Microbiol, Vol. 33, p. 1225.

A. O. Summers et S. Silver, (1978), Ann Rev Microbiol, Vol. 32, p. 637. M. THÉRÉNÉ, (1988). Thèse Doctorat Université Paris VI.

D. C. White, W. M. Davis, J. S. Nickels, J. D. King et R. J. Bobbie, (1979), CEcologia, Vol. 40, p. 51. 